\title{
Unperturbed Chain Dimension of Copolymers. I. Application of the Rotational Isomeric Method to Copolymers
}

\author{
Masahito OKA and Akio NAKAJIMA \\ Department of Polymer Chemistry, Kyoto University, \\ Sakyo-ku, Kyoto 606, Japan.
}

(Received January 7, 1978)

\begin{abstract}
The equation for the unperturbed mean-square radius of gyration $\left\langle S^{2}\right\rangle_{0}$ of copolymers was obtained for two cases by using the rotational isomeric method. For one case we assumed that the total mass of each structural unit of chain was situated on the skeletal atom. And for the other case we considered the deviation of the center of mass of each structual unit from each skeletal atom.

KEY WORDS Unperturbed Dimension / Radius of Gyration / Rotational Isomeric Method/Copolymer/Skeletal Atom/Structural Unit / Center of Mass / Long Side Chain /
\end{abstract}

Flory and his coworkers ${ }^{1,2}$ have derived the unperturbed chain dimension of real chains using the rotational isomeric model and information about the conformational energy for the skeletal bonds. Using this method, the unperturbed chain dimension was calculated for many kinds of real chains composed of one component. This method was applied to copolymers, and the mean-square end-to-end distance of unperturbed chains with independent rotation potentials, such as polypeptides, was calculated by Flory and his coworkers ${ }^{3}$ and by Tanaka and Nakajima. ${ }^{4}$ Flory and his coworkers calculated the composition dependence of the characteristic ratio $\left\langle R^{2}\right\rangle_{0} / n l^{2}$ for random copolypeptide chains composed of glycine, L-alanine, and D-alanine, and the structure dependence of $\left\langle R^{2}\right\rangle_{0} / n l^{2}$ for Markoffian copolypeptide chains composed of the same components. Tanaka and Nakajima ${ }^{4}$ carried out a similar calculation for copolypeptide chains composed of glycine, Lalanine, D-alanine, and their $N$-substituted amino acids.

Scholte ${ }^{5}$ has treated the unperturbed meansquare radius of gyration of block and graft copolymers using random flight statistics. However, the specificity of the individual real chains cannot be allowed for in his equations. The unperturbed mean-square radius of gyration of copolymers depend on the mass of the atoms which make up the chains, so they cannot be calculated by Flory's equation for simple chains. We tried to apply the rotational isomeric method to such copolymers, and obtained the equation for the unperturbed mean-square radius of gyration of copolymers for two cases. In one case the total mass of each structual unit of a chain is situated on the skeletal atom. In the other case there is a deviation of the center of mass of each structual unit from the skeletal atom because of the mass of the atoms on the long side chain.

\section{APPLICATION TO COPOLYMER CHAINS. I.}

A copolymer chain which is composed of $n+1$ skeletal atoms (structual units) is considered as a model. The mass of the $i$-th skeletal atoms is $a_{i}$. The vector from the $i$-th atom to the $j$-th atom is $\boldsymbol{r}_{i j}$. Scholte ${ }^{5}$ applied the theorem of Lagrange to this copolymer chain, and obtained eq 1 as the mean-square radius of gyration in the unperturbed state:

$$
\left\langle S^{2}\right\rangle_{0}=\left(1 / M^{2}\right) \sum_{0 \leq i<j \leq n} a_{i} a_{j}\left\langle r_{i j}^{2}\right\rangle_{0}
$$

where $M$ represents the total mass of the copolymer chain, i. e., $M=\sum_{i=0}^{n} a_{i}$. The suffix 0 in eq 1 indicates the unperturbed state. Using the rotational isomeric model, Flory found eq 2 for $\left\langle r_{i j}^{2}\right\rangle_{0}$ : 


$$
\left\langle r_{i j}^{2}\right\rangle_{0}=2 \boldsymbol{Z}^{-1} \boldsymbol{J}_{\nu}{ }^{*} \boldsymbol{U}_{1}^{(i)}\left[\begin{array}{ll}
\boldsymbol{E}_{\nu} & \mathbf{0}
\end{array}\right] \boldsymbol{A}_{i+1}^{(j-i)}\left[\begin{array}{c}
\mathbf{0} \\
\boldsymbol{E}_{\nu}
\end{array}\right] \boldsymbol{U}_{j+1}^{(n-j)} \boldsymbol{J}_{\nu}{ }^{\prime}
$$

where $Z$ is the partition function of this chain and is represented by the statistical weight matrix $\boldsymbol{U}_{i}$ commected with skeletal bond $i$.

$$
Z=J_{\nu}{ }^{*} \boldsymbol{U}_{1}{ }^{(n)} \boldsymbol{J}_{\nu}{ }^{\prime}
$$

$J_{\nu}{ }^{*}$ is defined as the row vector consisting of $\nu$ elements, the first element being unity and all the succeeding elements being zero. $\boldsymbol{J}_{\nu}{ }^{\prime}$ is defined as the column vector consisting of $\nu$ elements, all elements being unity. Symbolism of the type $\boldsymbol{A}_{i+1}^{(j-i)}$ (see ref 1) indicates the serial product of the type $A_{i+1} A_{i+2} \cdots A_{j} . O$ is the rectangular null matrix of the appropriate dimension. $\boldsymbol{E}_{\downarrow}$ is the unit matrix of the order $\nu . \quad A_{i}$ is the $5 \times 5$ matrix related to bond $i$, ans is represented by eq 3 :

$$
\boldsymbol{A}_{i}=\left[\begin{array}{ccc}
\boldsymbol{U} & \left(\boldsymbol{U} \otimes \boldsymbol{l}^{t}\right)\|\boldsymbol{T}\| & \left(\boldsymbol{l}^{2} / 2\right) \boldsymbol{U} \\
\mathbf{0} & \left(\boldsymbol{U} \otimes \boldsymbol{E}_{3}\right)\|\boldsymbol{T}\| & \boldsymbol{U} \otimes \boldsymbol{l} \\
\mathbf{0} & \mathbf{0} & \boldsymbol{U}
\end{array}\right]_{i}
$$

$\boldsymbol{l}_{i}{ }^{t}$ and $\boldsymbol{l}_{i}$ are the row and column vector from the $i$-1-th atom to the $i$-th atom, respectively; that is, the superscript $t$ of $l_{i}{ }^{t}$ means the transposed form of vector $l_{i}$. The original index $i$ appended to the bracket is understood to apply to the quantities within. $\boldsymbol{X} \otimes \boldsymbol{Y}$ represents the direct product between matrix $\boldsymbol{X}$ and $\boldsymbol{Y} . \quad\left\|\boldsymbol{T}_{i}\right\|$ is a pseudo diagonal matrix of order $3 \nu \otimes 3 \nu$, and is represented by the following equation:

$$
\left\|\boldsymbol{T}_{i}\right\|=\left[\begin{array}{ccc}
\boldsymbol{T}(1) & & \mathbf{0} \\
& \ddots & \\
\mathbf{0} & & \boldsymbol{T}(\nu)
\end{array}\right]_{i}
$$

where $\boldsymbol{T}_{i}(k)$ represents the coordinate transformation matrix when the rotational state of bond $i$ is $k$.

From eq 1 and 2, we obtain

$$
\begin{aligned}
\left\langle S^{2}\right\rangle_{0}= & (2 / M) Z^{-1} J_{\nu}{ }^{*} \sum_{0 \leq i<j \leq n} \sum_{1} U_{1}^{(i)}\left[\begin{array}{ll}
E_{\nu} & 0
\end{array}\right] a_{i} A_{i+1}^{(j-i)} \\
& \times\left[\begin{array}{c}
\mathbf{0} \\
\boldsymbol{E}_{\nu}
\end{array}\right] a_{j} \boldsymbol{U}_{j+1}^{(n-j)} \boldsymbol{J}_{\nu}{ }^{\prime}
\end{aligned}
$$

Representing matrix $\boldsymbol{P}_{i}$ and $\boldsymbol{Q}_{j}$ by the following equations,

$$
\begin{aligned}
& \boldsymbol{P}_{i}=\left[\begin{array}{ll}
\boldsymbol{E}_{\nu} & \mathbf{0}
\end{array}\right] a_{i} \\
& \boldsymbol{Q}_{j}=\left[\begin{array}{c}
\mathbf{0} \\
\boldsymbol{E}_{\nu}
\end{array}\right] a_{j}
\end{aligned}
$$

eq 4 is expressed as follows:

$$
\begin{aligned}
& \left\langle S^{2}\right\rangle_{0} \\
& \quad=\left(2 / M^{2}\right) Z^{-1} J_{\nu}{ }^{*} \sum_{0 \leq i<j \leq n} \sum_{1} U^{(i)} P_{i} A_{i+1}^{(j-i)} Q_{j} U_{j+1}^{(n-j)} J_{\nu}{ }^{\prime}
\end{aligned}
$$

As matrices $\boldsymbol{P}$ and $\boldsymbol{Q}$ have indices $i$ and $j$, respectively, the double sum in eq 5 cannot be summed in the same manner as for a simple chain. But, using eq 6 instead of eq 52 of Chapter 4 in ref 1 , we can obtain the double sum:

$$
\left[\begin{array}{ll}
\boldsymbol{E}_{\nu} & \mathbf{0}
\end{array}\right] \boldsymbol{F}_{0}{ }^{(n+1)}\left[\begin{array}{c}
\mathbf{0} \\
\boldsymbol{E}_{\nu}
\end{array}\right]
$$

where

$$
\boldsymbol{F}_{i}=\left[\begin{array}{ccc}
\boldsymbol{U} & \boldsymbol{U P} & \mathbf{0} \\
\mathbf{0} & \boldsymbol{A} & \boldsymbol{A} \boldsymbol{Q} \\
\mathbf{0} & \mathbf{0} & \boldsymbol{U}
\end{array}\right]_{i}
$$

We cannot define the state of rotation for the 1st skeletal bond and the $n$-th skeletal bond, so $\boldsymbol{U}_{1}=\boldsymbol{U}_{n}=\boldsymbol{E}_{\nu}$. The 0 -th skeletal bond does not exist, so the rotational state of that bond cannot be defined. But, for convenience's sake, we define $\boldsymbol{U}_{0}=\boldsymbol{E}_{\nu}$. Substituting eq 7 into eq 5 , we obtain

$$
\left\langle S^{2}\right\rangle_{0}=\left(2 / M^{2}\right) Z^{-1} J_{7 \nu}^{*} F_{0}{ }^{(n+1)} J_{7, \nu}^{\#}
$$

where $\boldsymbol{J}_{k, \nu}^{\sharp}$ is the column vector comprising $(k-1) \nu$ zeros followed by $\nu$ elements equal to unity. We rewrite eq 7 to the following equation:

$$
\boldsymbol{F}_{i}=\left[\begin{array}{ccccc}
\boldsymbol{U} & a \boldsymbol{U} & \mathbf{0} & \mathbf{0} & \mathbf{0} \\
\mathbf{0} & \boldsymbol{U} & \left(\boldsymbol{U} \otimes l^{t}\right)\|\boldsymbol{T}\| & \left(l^{2} / 2\right) \boldsymbol{U} & \left(l^{2} / 2\right) a \boldsymbol{U} \\
\mathbf{0} & \mathbf{0} & \left(\boldsymbol{U} \otimes \boldsymbol{E}_{3}\right)\|\boldsymbol{T}\| & \boldsymbol{U} \otimes \boldsymbol{l} & a \boldsymbol{U} \otimes \boldsymbol{l} \\
\mathbf{0} & \mathbf{0} & \mathbf{0} & \boldsymbol{U} & a \boldsymbol{U} \\
\mathbf{0} & \mathbf{0} & \mathbf{0} & \mathbf{0} & \boldsymbol{U}
\end{array}\right]_{i}
$$

The matrix $F_{i}$ contains all the required information relating to skeletal bond $i$ and skeletal unit $i$. By eq 8 , we can calculate the unperturbed dimension of a copolymer chain whose sequential distribution is known.

For the case of independent rotational chains, the rotation of a skeletal bond does not depend on the rotation of its adjacent bonds, so that we obtain the following simple results:

$$
\left\langle S^{2}\right\rangle_{0}=\left(2 / M^{2}\right) J_{7}{ }^{*} G_{0}{ }^{(n+1)} J_{7}
$$

where 


\section{Application to Copolymer Chains. I.}

$$
\boldsymbol{G}_{i}=\left[\begin{array}{ccccc}
1 & a & \mathbf{0} & 0 & 0 \\
0 & 1 & \boldsymbol{l}^{t}\langle\boldsymbol{T}\rangle & \boldsymbol{l}^{2} / 2 & \left(\boldsymbol{l}^{2} / 2\right) a \\
\mathbf{0} & \mathbf{0} & \langle\boldsymbol{T}\rangle & \boldsymbol{l} & a \boldsymbol{l} \\
0 & 0 & \mathbf{0} & 1 & a \\
0 & 0 & \mathbf{0} & 0 & 1
\end{array}\right]_{i}
$$

Matrix $\left\langle\boldsymbol{T}_{i}\right\rangle$ is the statistical average of the matrix $\boldsymbol{T}_{i}$, where the statistical average is carried out for the rotational states of skeletal bond $i$.

\section{APPLICATION TO COPOLYMER CHAINS. II.}

A copolymer chain which is composed of $n+1$ statistical units is considered as a model. The structural unit $i$ is composed of $p_{i}+1$ atoms. The skeletal bond of the structural unit $i$ is represented by $(i, 0)$, and the other atoms of it are represented by $(i, 1), \cdots,\left(i, p_{i}\right)$, respectively. The center of mass of structural unit $i$ is $G_{i}{ }^{*}$. The vectors from the skeletal atom $(i, 0)$ to $G_{i}{ }^{*}$ and to atom $(i, k)$ are respectively, $\boldsymbol{l}_{i}{ }^{*}$ and $\boldsymbol{r}_{i, k}^{*}$. The mass of atom $(i, k)$ is $a_{i, k}$, and the mass of structural unit $i$ is $a_{i}$. Then,

$$
a_{i}=\sum_{k=1}^{p_{i}+1} a_{i, k} .
$$

Applying the theorem of Lagrange to this copolymer chain, we obtain eq 12 as the mean-square radius of gyration in the unperturbed state. (See Appendix.)

$$
\begin{aligned}
\left\langle S^{2}\right\rangle_{0}= & (1 / M)^{2} \sum_{0 \leq i<j \leq n} a_{i} a_{j}\left\langle r_{i j}^{2}\right\rangle \\
& +\left(2 / M^{2}\right) \sum_{0 \leq i<j \leq n} a_{i} a_{j}\left\langle\boldsymbol{r}_{i j} \cdot\left(\boldsymbol{l}_{j}{ }^{*}-\boldsymbol{l}_{i}{ }^{*}\right)\right\rangle \\
& -\left(2 / M^{2}\right) \sum_{0 \leq i<j \leq n} a_{i} a_{j}\left\langle\boldsymbol{l}_{i}{ }^{*} \cdot \boldsymbol{l}_{j}{ }^{*}\right\rangle \\
& +(1 / M) \sum_{i=0}^{n} \sum_{k=0}^{p_{i}} a_{i, k}\left\langle r_{i, k}^{* 2}\right\rangle
\end{aligned}
$$

The first term of eq 12 corresponds to eq 1 , that is the equation based on the assumption that the total mass of the structural unit is situated on the skeletal atom. The 2nd, 3rd, and 4-th terms are the revised terms caused by the mass of the atoms on the long side chain. The 2 nd term of eq 12 is rewritten into

$$
\begin{aligned}
& \left(2 / M^{2}\right) \sum_{0 \leq i<j \leq n} \sum_{i} a_{i} a_{j}\left\langle\boldsymbol{r}_{i j} \cdot \boldsymbol{l}_{j}^{*}\right\rangle \\
& \quad-\left(2 / M^{2}\right) \sum_{0 \leq i<j \leq n} a_{i} a_{j}\left\langle\boldsymbol{r}_{i j} \cdot \boldsymbol{l}_{i}^{*}\right\rangle .
\end{aligned}
$$

We represent the first term of the former equation by $b_{1}$, and the second term by $-b_{2}$, for convenience's sake.

The term $b_{1}$ is obtained by the following method. When the rotational states of bond $j$ and bond $j+1$ are, respectively, $\xi$ and $\zeta$, we represent $l_{j}{ }^{*}$ by $l_{j, \xi \zeta}^{*}$. The statistical mechanical average of $l_{j}{ }^{*}$ over all configurations of the side chain is represented by $\overline{\boldsymbol{l}}_{j, \xi \zeta}^{*}$. And we defined $\overline{\boldsymbol{L}}_{j}{ }^{*}$ by eq 13 :

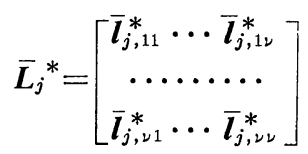

$\boldsymbol{l}_{j}{ }^{*}$ is expressed in the coordinate system of the bond $j+1$. Then, $\left\langle\boldsymbol{l}_{k} \cdot \boldsymbol{l}_{j}{ }^{*}\right\rangle$ is represented by the following equation:

$$
\begin{aligned}
& \left\langle\boldsymbol{l}_{k} \cdot \boldsymbol{l}_{j}{ }^{*}\right\rangle=\boldsymbol{Z}^{-1} \boldsymbol{J}_{\nu}{ }^{*} \boldsymbol{U}_{1}^{(k-1)}\left(\boldsymbol{E}_{\nu} \otimes \boldsymbol{l}_{k}{ }^{t}\right) \\
& \quad \times\left[\left(\boldsymbol{U} \otimes \boldsymbol{E}_{3}\right)\|\boldsymbol{T}\|\right]_{k}{ }^{(j-k+1)}\left[\overline{\boldsymbol{L}}_{j}{ }^{*} \odot \boldsymbol{U}_{j+1}\right] \boldsymbol{U}_{j+2}^{(n-j-1)} \boldsymbol{J}_{\nu}{ }^{\prime}
\end{aligned}
$$

where

$$
\left[\overline{\boldsymbol{L}}_{j} \odot \boldsymbol{U}_{j+1}\right]=\left[\begin{array}{lll}
\overline{\boldsymbol{l}}_{j, 11}^{*} u_{j+1,11} & \cdots & \bar{l}_{j, 1 \nu}^{*} u_{j+1,1 \nu} \\
\bar{l}_{j, \nu 1}^{*} u_{j+1, \nu 1} & \cdots & \overline{\boldsymbol{l}}_{j, \nu \nu}^{*} u_{j+1, \nu \nu}
\end{array}\right]
$$

Using the relation between $\boldsymbol{r}_{i j}$ and $\boldsymbol{l}_{k}$, i.e., $\boldsymbol{r}_{i j}=$ $\sum_{k=i+1}^{j} \boldsymbol{l}_{k},\left\langle\boldsymbol{r}_{i j} \cdot \boldsymbol{l}_{j}{ }^{*}\right\rangle$ is represented by the following equation:

$$
\begin{aligned}
& \left\langle\boldsymbol{r}_{i j} \cdot \boldsymbol{l}_{j}{ }^{*}\right\rangle=\sum_{k=i+1}^{j} \boldsymbol{Z}^{-1} \boldsymbol{J}_{\nu}{ }^{*} \boldsymbol{U}_{1}^{(k-1)}\left(\boldsymbol{E}_{\nu} \otimes \boldsymbol{l}_{k}{ }^{t}\right) \\
& \quad \times\left[\left(\boldsymbol{U} \otimes \boldsymbol{E}_{3}\right)\|\boldsymbol{T}\|\right]_{k}{ }^{(j-k+1)}\left[\overline{\boldsymbol{L}}_{j}{ }^{*} \odot \boldsymbol{U}_{j+1}\right] \boldsymbol{U}_{j+2}^{(n-j-1)} \boldsymbol{J}_{\nu}{ }^{\prime}
\end{aligned}
$$

The sum in this equation is obtained by using the following matrix, $\boldsymbol{H}_{\boldsymbol{i}}$ :

$$
\boldsymbol{H}_{i}=\left[\begin{array}{ll}
\boldsymbol{U} & \left(\boldsymbol{U} \otimes \boldsymbol{l}^{t}\right)\|\boldsymbol{T}\| \\
\mathbf{0} & \left(\boldsymbol{U} \otimes \boldsymbol{E}_{3}\right)\|\boldsymbol{T}\|
\end{array}\right]_{i}
$$

Thus,

$$
\begin{aligned}
\left\langle\boldsymbol{r}_{i j} \cdot \boldsymbol{l}_{j}{ }^{*}\right\rangle= & \boldsymbol{Z}^{-1} \boldsymbol{J}_{\nu}{ }^{*} \boldsymbol{U}_{1}^{(i)}\left[\begin{array}{ll}
\boldsymbol{E}_{\nu} & \mathbf{0}
\end{array}\right] \boldsymbol{H}_{i+1}^{(j-i)}\left[\begin{array}{c}
\mathbf{0} \\
\boldsymbol{E}_{3}
\end{array}\right] \\
& \times\left[\overline{\boldsymbol{L}}_{j}{ }^{*} \odot \boldsymbol{U}_{j+1}\right] \boldsymbol{U}_{j+2}^{(n-j-1)} \boldsymbol{J}_{\nu}{ }^{\prime}
\end{aligned}
$$

Using this equation, we obtain $b_{1}$ :

$$
b_{1}=\left(2 / M^{2}\right) Z^{-1} J_{6 \nu}^{*} K_{0}^{(n+1)} J_{6, \nu}^{\#}
$$

where 


$$
\boldsymbol{K}_{i}=\left[\begin{array}{ccc}
\boldsymbol{U}_{i} & \boldsymbol{a}_{i} \boldsymbol{U}_{i} & \mathbf{0} \\
\mathbf{0} & \boldsymbol{U}_{i} & \left(\boldsymbol{U}_{i} \otimes \boldsymbol{l}_{i}{ }^{* t}\right)\left\|\boldsymbol{T}_{i}\right\| \\
\mathbf{0} & \mathbf{0} & \left(\boldsymbol{U}_{i} \otimes \boldsymbol{E}_{3}\right)\left\|\boldsymbol{T}_{i}\right\| \\
\mathbf{0} & \mathbf{0} & \mathbf{0}
\end{array}\right.
$$

Then, the term $b_{2}$ is obtained by the following method. We define matrix $\overline{\boldsymbol{N}}_{j}^{*}$ by eq 18 :

$$
\bar{N}_{j}^{*}=\left[\begin{array}{ccc}
\overline{\boldsymbol{l}}_{j, 11}^{* t} & \cdots & \overline{\boldsymbol{l}}_{j, 1 \nu}^{* t} \\
\cdots & \cdots & \cdots \\
\overline{\boldsymbol{l}}_{j, \nu 1}^{* t} & \cdots & \overline{\boldsymbol{l}}_{j, \nu \nu}^{* t}
\end{array}\right]
$$

Using eq 18 , we have

$$
\begin{aligned}
\left\langle\boldsymbol{l}_{i}{ }^{*} \cdot \boldsymbol{r}_{i j}\right\rangle & =\boldsymbol{Z}^{-1} \boldsymbol{J}_{\nu}{ }^{*} \boldsymbol{U}_{1}{ }^{(i)}\left[\overline{\boldsymbol{N}}_{i}{ }^{*} \odot \boldsymbol{U}_{i+1}\right]\left(\boldsymbol{E}_{\nu} \otimes \boldsymbol{l}_{i+1}\right) \\
& \times \boldsymbol{U}_{i+2}^{(n-i-1)} \boldsymbol{J}_{\nu}{ }^{\prime}+\sum_{k=i+2}^{j} \boldsymbol{Z}^{-1} \boldsymbol{J}_{\nu}{ }^{*} \boldsymbol{U}_{1}{ }^{(i)} \\
& \times\left[\overline{\boldsymbol{N}}_{i}{ }^{*} \odot \boldsymbol{U}_{i+1}\right]\left\|\boldsymbol{T}_{i+1}\right\|\left[\left(\boldsymbol{U} \otimes \boldsymbol{E}_{3}\right)\|\boldsymbol{T}\|\right]_{i+2}^{(k-i-2)} \\
& \times\left(\boldsymbol{E}_{\nu} \otimes \boldsymbol{l}_{k}\right) \boldsymbol{U}_{k}{ }^{(n-k+1)} \boldsymbol{J}_{\nu}{ }^{\prime}
\end{aligned}
$$

We define

$$
\boldsymbol{W}_{i}=\left[\begin{array}{cc}
\boldsymbol{U}_{i} & \boldsymbol{U}_{i}\left[\overline{\boldsymbol{N}}_{i}^{*} \odot \boldsymbol{U}_{i+1}\right]\left\|\boldsymbol{T}_{i+1}\right\| \\
\mathbf{0} & \left(\boldsymbol{U}_{i+1} \otimes \boldsymbol{E}_{3}\right)\left\|\boldsymbol{T}_{i+1}\right\| \\
\mathbf{0} & \mathbf{0} \\
\mathbf{0} & \mathbf{0}
\end{array}\right.
$$

The 3rd term of eq 12 is obtained by the following method. Then, we have

$$
\begin{aligned}
& \left\langle\boldsymbol{l}_{i}{ }^{*} \cdot \boldsymbol{l}_{j}{ }^{*}\right\rangle=\boldsymbol{Z}^{-1} \boldsymbol{J}_{\nu}{ }^{*} \boldsymbol{U}_{1}^{(i)}\left[\overline{\boldsymbol{N}}_{i}{ }^{*} \odot \boldsymbol{U}_{i+1}\right]\left\|\boldsymbol{T}_{i+1}\right\| \\
& \quad \times\left[\left(\boldsymbol{U} \otimes \boldsymbol{E}_{3}\right)\|\boldsymbol{T}\|\right]_{i+2}^{(j-i-1)}\left[\overline{\boldsymbol{L}}_{j}{ }^{*} \odot \boldsymbol{U}_{j+1}\right] \boldsymbol{U}_{j+2}^{(n-j-1)} \boldsymbol{J}_{\nu}{ }^{\prime}
\end{aligned}
$$

where we have defined $\left[\left(\boldsymbol{U} \otimes \boldsymbol{E}_{3}\right)\|\boldsymbol{T}\|\right]_{i+2}^{(0)}=\boldsymbol{E}_{3 \nu}$ for simplicity in expression. Using this equation, we obtain

$$
\begin{aligned}
\left(2 / \boldsymbol{M}^{2}\right) & \sum_{0 \leq i<j \leq n} a_{i} a_{j}\left\langle\boldsymbol{l}_{i}{ }^{*} \boldsymbol{l}_{j}{ }^{*}\right\rangle \\
= & \left(2 / \boldsymbol{M}^{2}\right) \sum_{0 \leq i<j \leq n} \sum^{-1} \boldsymbol{J}_{\nu}{ }^{*} \boldsymbol{U}_{1}^{(i)} \boldsymbol{a}_{i}\left[\overline{\boldsymbol{N}}_{i}{ }^{*} \odot \boldsymbol{U}_{i+1}\right] \\
& \times\left\|\boldsymbol{T}_{i+1}\right\|\left[\left(\boldsymbol{U} \otimes \boldsymbol{E}_{3}\right)\|\boldsymbol{T}\|\right]_{i+2}^{(j-i-1)} \\
& \times \boldsymbol{a}_{j}\left[\overline{\boldsymbol{L}}_{j}{ }^{*} \odot \boldsymbol{U}_{j+1}\right] \boldsymbol{U}_{j+2}^{(n-j-1)} \boldsymbol{J}_{\nu}{ }^{\prime}
\end{aligned}
$$

Using matrix $C_{i}$, the double sum of eq 24 is also obtained. Thus,

$$
\begin{aligned}
& \left(2 / M^{2}\right) \sum_{0 \leq i<j \leq n} a_{i} a_{j}\left\langle\boldsymbol{l}_{i}{ }^{*} \boldsymbol{l}_{j}{ }^{*}\right\rangle \\
& =\left(2 / M^{2}\right) Z^{-1} \boldsymbol{J}_{5 \nu}{ }^{*} \boldsymbol{C}_{\mathbf{0}}{ }^{(n+1)} \boldsymbol{J}_{5, \nu}^{\sharp}
\end{aligned}
$$

where

$$
\begin{aligned}
& \left.\begin{array}{c}
\mathbf{0} \\
a_{i}\left(\boldsymbol{U}_{i} \otimes \boldsymbol{l}_{i}^{* t}\right)\left\|\boldsymbol{T}_{i}\right\|\left[\overline{\boldsymbol{L}}_{i}^{*} \odot \boldsymbol{U}_{i+1}\right] \\
a_{i}\left(\boldsymbol{U}_{i} \otimes \boldsymbol{E}_{3}\right)\left\|\boldsymbol{T}_{i}\right\|\left[\overline{\boldsymbol{L}}_{i}^{*} \odot \boldsymbol{U}_{i+1}\right] \\
\boldsymbol{U}_{i+1}
\end{array}\right] \\
& \boldsymbol{V}_{k}{ }^{\prime}=\left[\begin{array}{cc}
\|\boldsymbol{T}\| & \boldsymbol{E}_{\nu} \otimes \boldsymbol{l} \\
\mathbf{0} & \boldsymbol{U}
\end{array}\right]_{k} \\
& \boldsymbol{V}_{k}=\left[\begin{array}{cc}
\left(\boldsymbol{U} \otimes \boldsymbol{E}_{3}\right)\|\boldsymbol{T}\| & \left(\boldsymbol{E}_{\nu} \otimes \boldsymbol{l}\right) \boldsymbol{U} \\
\mathbf{0} & \boldsymbol{U}
\end{array}\right]_{k}
\end{aligned}
$$

Substituting eq 20 and 21 into eq 19 , we obtain

$$
\begin{aligned}
\left\langle\boldsymbol{l}_{i}{ }^{*} \cdot \boldsymbol{r}_{i j}\right\rangle= & Z^{-1} \boldsymbol{J}_{\nu}{ }^{* t} \boldsymbol{U}_{1}^{(i)}\left[\overline{\boldsymbol{N}}_{i}{ }^{*} \odot \boldsymbol{U}_{i+1}\right]\left[\begin{array}{ll}
\boldsymbol{E}_{3} & 0
\end{array}\right] \\
& \times \boldsymbol{V}_{i+1}^{\prime} \boldsymbol{V}_{i+2}^{(j-i-1)}\left[\begin{array}{c}
\mathbf{0} \\
\boldsymbol{E}_{\nu}
\end{array}\right] \boldsymbol{U}_{j+1}^{(n-j)} \boldsymbol{J}_{\nu}{ }^{\prime}
\end{aligned}
$$

Thus, using this equation, we obtain

$$
b_{2}=\left(2 / M^{2}\right) Z^{-1} J_{6 \nu}^{*} W_{0}^{(n+)} J_{6, \nu}^{\#}
$$

where

$$
\left.\begin{array}{cc}
\boldsymbol{U}_{i}\left[\overline{\boldsymbol{N}}_{i}^{*} \otimes \boldsymbol{U}_{i+1}\right]\left(\boldsymbol{E}_{\nu} \otimes \boldsymbol{l}_{i+1}\right) & \mathbf{0} \\
\left(\boldsymbol{E}_{\nu} \otimes \boldsymbol{l}_{i+1}\right) \boldsymbol{U}_{i+1} & \mathbf{0} \\
\boldsymbol{U}_{i+1} & a_{i} \boldsymbol{E}_{\nu} \\
\mathbf{0} & \boldsymbol{U}_{i}
\end{array}\right]
$$

$$
\boldsymbol{C}_{i}=\left[\begin{array}{ccc}
\boldsymbol{U}_{i} & \boldsymbol{U}_{i} a_{i}\left[\overline{\boldsymbol{N}}_{i}^{*} \odot \boldsymbol{U}_{i+1}\right]\left\|\boldsymbol{T}_{i+1}\right\| & \mathbf{0} \\
\mathbf{0} & \left(\boldsymbol{U}_{i+1} \otimes \boldsymbol{E}_{3}\right)\left\|\boldsymbol{T}_{i+1}\right\| & a_{i}\left[\overline{\boldsymbol{L}}_{i} \odot \boldsymbol{U}_{i+1}\right] \\
\mathbf{0} & \mathbf{0} & \boldsymbol{U}_{i+1}
\end{array}\right]
$$

The vector $l_{j, \xi \zeta}^{*}$ in eq 13 and the last term of eq 12 can be calculated by a simple method, if the structure of the side chain is known. Thus, using eq $8,16,22$, and 25 , eq 12 is rewritten to give the final result, eq 27.

$$
\begin{aligned}
\left\langle S^{2}\right\rangle_{0}= & \left(2 / M^{2}\right) Z^{-1} J_{7}{ }^{*} \boldsymbol{F}_{0}{ }^{(n+1)} \boldsymbol{J}_{7, \nu}^{\sharp} \\
& -\left(2 / M^{2}\right) Z^{-1} J_{6}{ }^{*} \boldsymbol{W}_{0}^{(n+1)} \boldsymbol{J}_{6, \nu}^{\sharp} \\
& +\left(2 / \boldsymbol{M}^{2}\right) \boldsymbol{Z}^{-1} \boldsymbol{J}_{6}{ }^{*} \boldsymbol{K}_{0}{ }^{(n+1)} \boldsymbol{J}_{6, \nu}^{\sharp} \\
& -\left(2 / \boldsymbol{M}^{2}\right) \boldsymbol{Z}^{-1} \boldsymbol{J}_{5}{ }^{*} \boldsymbol{C}_{0}{ }^{(n+1)} \boldsymbol{J}_{5, \nu}^{\sharp} \\
& +(1 / \boldsymbol{M}) \sum_{i=0}^{n} \sum_{k=0}^{p_{i}} a_{i, k}\left\langle r_{i, k}^{* 2}\right\rangle
\end{aligned}
$$

Then, we calculate $\bar{l}_{j, \xi \zeta}^{*}$ and the last term of eq 12 using a model of structural units. This model is shown in Figure 1. We assume that a structural 


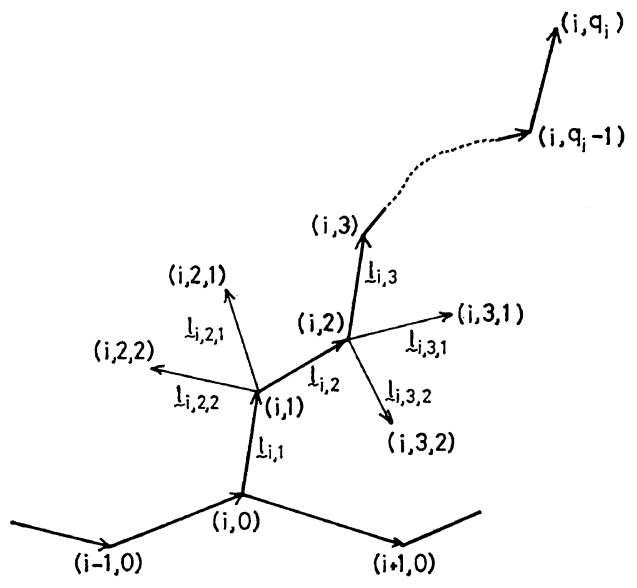

Figure 1. A side chain model which has no branching composed of more than two bonds.

unit has a long side chain and that the side chain has no branching composed of more than two bonds. These assumptions are very reasonable for analysing real chains such as vinyl polymers and polypeptides. The skeletal bonds of side chain $i$ are represented by symbols $(i, 1), \cdots$, and $\left(i, q_{i}\right)$ towards the end of the side chain, respectively. And the atoms attached to the skeletal atom $(i, h-1)$ of side chain $i$ is represented by symbols $(i, h, 0), \cdots$, and $\left(i, h, \alpha_{i, h}\right)$. For real chains, $\alpha_{i, h}$ takes on the value 0,1 , or 2 . The atom $(i, h, 0)$ is the skeletal atom $(i, h)$ of the side chain. Thus, we obtain the relation

$$
p_{i}=\sum_{h=1}^{q_{i}}\left(\alpha_{i, h}+1\right)
$$

between $p_{i}$ and $q_{i}$. The vectors from the atom $(i, h-1)$ to the atom $(i, h, g)$ and to the atom $(i, h)$ are, respectively, $\boldsymbol{l}_{i, h, g}$ and $\boldsymbol{l}_{i, h}$. The vector from the atom $(i, 0)$ to the atom $(i, h, g)$ is $r_{i, h, g}^{*}$. The mass of atom $(i, h, g)$ is $a_{i, h, g}$, and $a_{i, h, 0}=a_{i, h}$. From eq A7, we obtain the side chain vector $\tilde{l}_{i, \xi \zeta}^{*}$,

$$
l_{i, \xi \zeta}^{*}=\left(1 / a_{i}\right) \sum_{k=1}^{q_{i}} \sum_{g=0}^{\alpha_{i, k}} a_{i, k, g} r_{i, k, g}^{*}
$$

where

$$
a_{i}=\sum_{k=1}^{q_{i}} \sum_{g=0}^{\alpha_{i, k}} a_{i, k, g}
$$

Then,

$$
r_{i, k, g}^{*}=\left(\sum_{h=1}^{k-1} \boldsymbol{l}_{i, h}\right)+\boldsymbol{l}_{i, k, g}
$$

We define $\boldsymbol{T}_{i, k}$ as the matrix which transforms a vector represented in the coordinate system of bond $(i, k+1)(i . e$., reference frame $(i, k+1))$ into that represented in the coordinate system of bond $(i, k)$. And $\boldsymbol{T}_{i, 0}$ is the transformation matrix from reference frame $(i, 1)$ to reference frame $i+1$. The bond vector $\boldsymbol{l}_{l, k, g}$ is represented in the coordinate system of bond $(i, k)$. Using the matrix $\boldsymbol{T}_{i, k}$, we obtain the following equation from eq 29:

$$
r_{i, k, g}^{*}=\left(\sum_{h=0}^{k-1} \boldsymbol{T}_{i, 0}^{(h)} \boldsymbol{l}_{i, h}\right)+\boldsymbol{T}_{i, 0}^{(k)} \boldsymbol{l}_{i, k, g}
$$

We represent the statistical weight matrix for the rotation about the bond $(i, h)$ by $\boldsymbol{V}_{i, h} . \quad \boldsymbol{V}_{i, h}$ depends on the states of bond $h-1$ and bond $h$. We define $\overline{\boldsymbol{r}}_{i, k, g}^{*}$ as $\boldsymbol{r}_{i, k, g}^{*}$ averaged over all configurations of the side chain. Then,

$$
\begin{aligned}
\overline{\boldsymbol{r}}_{i, k, g}^{*}= & \beta_{i}{ }^{-1}\left\{\sum_{h=1}^{k-1}\left(\boldsymbol{J}_{\nu}{ }^{*} \otimes \boldsymbol{T}_{i, 0}\right)\left[\boldsymbol{V}_{\boldsymbol{i}}, \otimes \boldsymbol{E}_{3}\right)\left\|\boldsymbol{T}_{i,}\right\|\right]_{1}^{(h-1)} \\
& \times\left(\boldsymbol{E}_{\nu} \otimes \boldsymbol{I}_{i, h}\right) \boldsymbol{V}_{i, h}^{\left(q_{i}-h+1\right)} \boldsymbol{J}_{\nu}{ }^{\prime} \\
& +\left(\boldsymbol{J}_{\nu}{ }^{*} \otimes \boldsymbol{T}_{i, 0}\right)\left[\left(\boldsymbol{V}_{i,} \otimes \boldsymbol{E}_{3}\right)\left\|\boldsymbol{T}_{i,}\right\|\right]_{1}^{(k-1)} \\
& \left.\times\left(\boldsymbol{E}_{\nu} \otimes \boldsymbol{I}_{i, k, g}\right) \boldsymbol{V}_{i, k}^{\left(q_{i}-k+1\right)} \boldsymbol{J}_{\nu}{ }^{\prime}\right\}
\end{aligned}
$$

where

$$
\beta_{i}=\boldsymbol{J}_{\nu}{ }^{*} V_{1}{ }^{\left(q_{i}\right)} \boldsymbol{J}_{\nu}{ }^{\prime}
$$

Using eq 28 and 30 , we obtain $\overline{\boldsymbol{l}}_{j}{ }^{*}$,

$$
\begin{aligned}
\overline{\boldsymbol{l}}_{i, \xi \zeta}^{*}= & \left(1 / \boldsymbol{a}_{i}\right) \beta_{i}{ }^{-1}\left\{\sum_{k=1}^{q_{i}} \boldsymbol{a}_{i, k}^{\prime} \sum_{h=1}^{k-1}\left(\boldsymbol{J}_{\nu}{ }^{*} \otimes \boldsymbol{T}_{i, 0}\right)\right. \\
& \times\left[\left(\boldsymbol{V}_{i,} \otimes \boldsymbol{E}_{3}\right) \| \boldsymbol{T}_{i, \|]_{1}{ }^{(h-1)}}\right. \\
& \times\left(\boldsymbol{E}_{\nu} \otimes \boldsymbol{l}_{i, h}\right) \boldsymbol{V}_{i, h}^{\left(q_{i}-h+1\right)} \boldsymbol{J}_{\nu}{ }^{\prime} \\
& +\sum_{k=1}^{q_{i}}\left(\boldsymbol{J}_{\nu}{ }^{*} \otimes \boldsymbol{T}_{i, 0}\right)\left[\left(\boldsymbol{V}_{i}, \otimes \boldsymbol{E}_{3}\right)\left\|\boldsymbol{T}_{i}\right\|\right]_{1}{ }^{(k-1)} \\
& \left.\times \boldsymbol{L}_{i, k} \boldsymbol{V}_{i, k+1}^{\left(q_{i-k}\right)} \boldsymbol{J}_{\nu}{ }^{\prime}\right\}
\end{aligned}
$$

where

$$
\begin{aligned}
\boldsymbol{a}_{i, k}^{\prime} & =\sum_{g=0}^{\alpha_{i, k}} a_{i, k, g} \\
\boldsymbol{L}_{i, k} & =\sum_{g=0}^{\alpha_{i, k}} a_{i, k, g}\left(\boldsymbol{V}_{i, k} \otimes \boldsymbol{l}_{i, k, g}\right)
\end{aligned}
$$

The summation about $k$ and $h$ in eq 31 is obtained by matrices $\boldsymbol{G}_{i, k}$ and $\boldsymbol{H}_{i, h}$ :

$$
\begin{aligned}
& \overline{\boldsymbol{l}}_{i, \xi \zeta}^{*}=\left(1 / a_{i}\right) \beta_{i}^{-1}\left[\begin{array}{ll}
\boldsymbol{T}_{i, 0} & \mathbf{0}
\end{array}\right] \boldsymbol{G}_{i, 1}^{\left(\boldsymbol{q}_{i}\right)} \boldsymbol{J}_{5, \nu}^{\#} \\
& +\left[\begin{array}{ll}
\boldsymbol{T}_{i, 0} & \mathbf{0}
\end{array}\right] \boldsymbol{H}_{i, 1}^{\left(q_{i}\right)} \boldsymbol{J}_{4, \nu}^{\#}
\end{aligned}
$$


where

$$
\begin{aligned}
\boldsymbol{G}_{i, k} & =\left[\begin{array}{ccc}
\left(\boldsymbol{V} \otimes \boldsymbol{E}_{3}\right)\|\boldsymbol{T}\| & (\boldsymbol{V} \otimes \boldsymbol{l}) & \mathbf{0} \\
\mathbf{0} & \boldsymbol{V} & \boldsymbol{a}^{\prime} \boldsymbol{V} \\
\mathbf{0} & \mathbf{0} & \boldsymbol{V}
\end{array}\right]_{i, k} \\
\boldsymbol{H}_{i, k} & =\left[\begin{array}{cc}
\left(\boldsymbol{V} \otimes \boldsymbol{E}_{3}\right)\|\boldsymbol{T}\| & \boldsymbol{L} \\
\mathbf{0} & \boldsymbol{V}
\end{array}\right]_{i, k}
\end{aligned}
$$

The last term of eq 12 is obtained by the following method. Using eq $29, \overline{\boldsymbol{r}}_{i, k}^{* 2}, g$ is represented by

$$
\begin{aligned}
\overline{\boldsymbol{r}}_{i, k, g}^{* 2}= & \sum_{h=1}^{k-1}\left\langle\boldsymbol{l}_{i, h}^{2}\right\rangle+2 \sum_{1 \leq e<f \leq l-1}\left\langle\boldsymbol{l}_{i, e} \boldsymbol{l}_{i, f}\right\rangle \\
& +2 \sum_{h=1}^{k-1}\left\langle\boldsymbol{l}_{i, h} \boldsymbol{l}_{i, k, g}\right\rangle+\boldsymbol{l}_{i, k, g}^{2}
\end{aligned}
$$

Substituting matrices $\boldsymbol{C}_{i, k}$ and $\boldsymbol{I}_{i, k}$ into this equation, we obtain the following equation:

$$
\begin{aligned}
\tilde{\boldsymbol{r}}_{i, k, g}^{* 2}= & 2 \beta_{i}{ }^{-1} \boldsymbol{J}_{5}{ }^{*} \boldsymbol{C}_{i, 1}^{(k-1)}\left[\begin{array}{c}
\mathbf{0} \\
\boldsymbol{E}_{\nu}
\end{array}\right] \boldsymbol{V}_{i, k}^{\left(q_{i}-k+1\right)} \boldsymbol{J}_{\nu}{ }^{\prime} \\
& +2 \beta_{i}{ }^{-1} \boldsymbol{J}_{4}{ }^{*} \boldsymbol{I}_{i, 1}^{(k-1)}\left[\begin{array}{c}
\mathbf{0} \\
\boldsymbol{E}_{3 \nu}
\end{array}\right]\left(\boldsymbol{E}_{\nu} \otimes \boldsymbol{l}_{i, k, g}\right) \\
& \times \boldsymbol{V}_{i, k}^{\left(q_{i}-k+1\right)} \boldsymbol{J}_{\nu}{ }^{\prime}+\boldsymbol{l}_{i, k, g}^{2}
\end{aligned}
$$

where

$$
\begin{aligned}
\boldsymbol{C}_{i, k} & =\left[\begin{array}{ccc}
\boldsymbol{V} & \left(\boldsymbol{V} \otimes \boldsymbol{l}^{\boldsymbol{t}}\right)\|\boldsymbol{T}\| & \left(\boldsymbol{l}^{2} / 2\right) \boldsymbol{V} \\
\mathbf{0} & \left(\boldsymbol{V} \otimes \boldsymbol{E}_{3}\right)\|\boldsymbol{T}\| & \boldsymbol{V} \otimes \boldsymbol{l} \\
\mathbf{0} & \mathbf{0} & \boldsymbol{V}
\end{array}\right]_{i, k} \\
\boldsymbol{I}_{i, k} & =\left[\begin{array}{cc}
\boldsymbol{V} & \left(\boldsymbol{V} \otimes \boldsymbol{l}^{\boldsymbol{t}}\right)\|\boldsymbol{T}\| \\
\mathbf{0} & \left(\boldsymbol{V} \otimes \boldsymbol{E}_{3}\right)\|\boldsymbol{T}\|
\end{array}\right]_{i, k}
\end{aligned}
$$

Then, we define $\bar{r}_{i, \xi \zeta}^{* 2}$ by the following equation:

$$
\bar{r}_{i, \xi \zeta}^{* 2}=\sum_{k=1}^{q_{i}} \sum_{g=0}^{\alpha_{i, k}} a_{i, k, g} \bar{r}_{i, k, g}^{* 2}
$$

Substituting eq 33 into this equation, we obtain

$$
\begin{aligned}
\overline{\boldsymbol{r}}_{i, \xi \zeta}^{* 2}= & 2 \beta_{i}{ }^{-1} \sum_{k=1}^{q_{i}} \boldsymbol{J}_{5}{ }^{*} \boldsymbol{C}_{i, 1}^{(k-1)}\left[\begin{array}{c}
\mathbf{0} \\
\boldsymbol{E}_{\nu}
\end{array}\right] \boldsymbol{a}_{i, k}^{\prime} \boldsymbol{V}_{i, k}^{\left(\boldsymbol{q}_{i}-k+1\right)} \boldsymbol{J}_{\nu}{ }^{\prime} \\
& +2 \beta_{i}{ }^{-1} \sum_{k=1}^{q_{i}} \boldsymbol{J}_{4}{ }^{*} \boldsymbol{I}_{i, 1}^{(k-1)}\left[\begin{array}{c}
\mathbf{0} \\
\boldsymbol{E}_{3 \nu}
\end{array}\right] \boldsymbol{L}_{i, k} \boldsymbol{V}_{i, k+1}^{\left(q_{i}-k\right)} \boldsymbol{J}_{\nu}{ }^{\prime} \\
& +\sum_{g=0}^{\alpha_{i, k}} a_{i, k, g} l_{i, k, g}^{2}
\end{aligned}
$$

Using matrices $\boldsymbol{M}_{i, k}$ and $\boldsymbol{R}_{i, k}$, the summation of this equation is obtained:

$$
\begin{aligned}
\overline{\boldsymbol{r}}_{i, \xi \zeta}^{* 2}= & 2 \beta_{i}{ }^{-1} \boldsymbol{J}_{6}{ }^{*} \boldsymbol{M}_{i, 1}^{\left(q_{i}\right)} \boldsymbol{J}_{6, \nu}^{\#}+2 \beta_{i}{ }^{-1} \boldsymbol{J}_{5}{ }^{*} \boldsymbol{R}_{i, 1}^{\left(q_{i}\right)} \boldsymbol{J}_{5, \nu}^{\#} \\
& +\sum_{k=1}^{q_{i}} \sum_{g=0}^{\alpha_{i, k}} a_{i, k, g} l_{i, k, g}^{2}
\end{aligned}
$$

where

$$
\begin{aligned}
\boldsymbol{M}_{i, k} & =\left[\begin{array}{cccc}
\boldsymbol{V} & \left(\boldsymbol{V} \otimes \boldsymbol{l}^{t}\right)\|\boldsymbol{T}\| & \left(\boldsymbol{l}^{2} / 2\right) \boldsymbol{V} & \mathbf{0} \\
\mathbf{0} & \left(\boldsymbol{V} \otimes \boldsymbol{E}_{3}\right)\|\boldsymbol{T}\| & \boldsymbol{V} \otimes \boldsymbol{l} & \mathbf{0} \\
\mathbf{0} & \mathbf{0} & \boldsymbol{V} & \boldsymbol{a}^{\prime} \boldsymbol{V} \\
\mathbf{0} & \mathbf{0} & \mathbf{0} & \boldsymbol{V}
\end{array}\right]_{i, k} \\
\boldsymbol{R}_{i, k} & =\left[\begin{array}{ccc}
\boldsymbol{V} & \left(\boldsymbol{V} \otimes \boldsymbol{l}^{t}\right)\|\boldsymbol{T}\| & \mathbf{0} \\
\mathbf{0} & \left(\boldsymbol{V} \otimes \boldsymbol{E}_{3}\right)\|\boldsymbol{T}\| & \boldsymbol{L} \\
\mathbf{0} & \mathbf{0} & \boldsymbol{V}
\end{array}\right]_{i, k}
\end{aligned}
$$

$\tilde{r}_{i, \xi \zeta}^{* 2}$ is the statistical mechanical average of $\tilde{r}_{i}^{* 2}$ over all configurations of side chains when the rotational states of bonds $j$ and $j+1$ are, respectively, $\xi$ and $\zeta$. The statistical average of $\bar{r}_{i}^{* 2}$ over all configuration of the main chain is obtained by the following equation:

$$
\left\langle{\overline{\boldsymbol{r}}_{i}}^{* 2}\right\rangle=Z^{-1} \boldsymbol{J}_{\nu}{ }^{*} \boldsymbol{U}_{1}^{(i-1)} \boldsymbol{D}_{i} \boldsymbol{U}_{i+1}^{(n-1)} \boldsymbol{J}_{\nu}{ }^{\prime}
$$

where

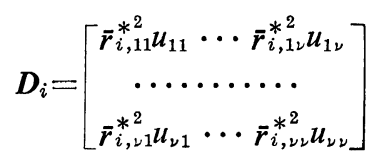

From the definition of $\left\langle\bar{r}_{i}^{* 2}\right\rangle$ of eq 35 and $\left\langle r_{i, k}^{* 2}\right\rangle$ of eq 12 , we obtain the following relation:

$$
\left\langle\overline{\boldsymbol{r}}_{i}^{* 2}\right\rangle=\sum_{k=0}^{p_{i}} a_{i, k}\left\langle r_{i, k}^{* 2}\right\rangle
$$

Using this equation, the last term of eq 12 is obtained.

$$
(1 / M) Z^{-1} \sum_{i=0}^{n} J_{\nu}{ }^{*} U_{0}^{(i)} D_{i} U_{i+1}^{(n-1)} J_{\nu}{ }^{\prime}
$$

The summation about $i$ of this equation is obtained by using the following matrix:

$$
\boldsymbol{B}_{i}=\left[\begin{array}{ll}
\boldsymbol{U} & \boldsymbol{D} \\
\mathbf{0} & \boldsymbol{U}
\end{array}\right]_{i}
$$

Finally, we obtain the following result:

$$
(1 / M) \sum_{i=0}^{n} \sum_{k=1}^{p_{i}} a_{i, k}\left\langle r_{i, k}^{* 2}\right\rangle=(1 / M) Z^{-1} J_{2 \nu}^{*} B_{0}{ }^{(n+1)} J_{2, \nu}^{\#}
$$

\section{DISCUSSION}

Recently, Mattice ${ }^{6}$ has obtained the meansquare radius of gyration in the unperturbed state of copolymer chains for the case in which the center 
Application to Copolymer Chains. I.

of mass of each structural unit is situated on each skeletal atom, i.e., case I of this paper. But his matrix $\boldsymbol{S}_{\boldsymbol{i}}$ is different from our matrices $\boldsymbol{F}_{\boldsymbol{i}}$ for chains with interdependent rotational potentials, and $\boldsymbol{G}_{i}$ for chains with independent rotational potentials. The elements in our matrix $\boldsymbol{G}_{i}$ take only suffix $i$, but those in his matrix $S_{i}$ take suffices $i-1$ and $i$. That is, his matrix $S_{i}$ depends on the characters of structural units $i-1$ and $i$. The calculation of $\left\langle S^{2}\right\rangle_{0}$ is thus very complicated when $\boldsymbol{S}_{i}$ is used. For the calculation for a copolymer chain composed of two components, the matrix $\boldsymbol{S}_{i}$ has to be distinguished into four kinds depending on the combination of the two components, but our matrix $\boldsymbol{G}_{i}$ only into two kinds corresponding to the respective components.

We have used $a_{i}$ for the mass of structural unit $i$ for our equations. But we can use $a_{i}$ for other quantities. For example, $\left\langle S^{2}\right\rangle_{0}$ obtained by the light scattering method ${ }^{7-10}$ is not $\left\langle S^{2}\right\rangle_{0}$ based on the mass of the structural unit, but $\left\langle S^{2}\right\rangle_{0}$ based on the polarizability. For a chain composed of one component, these two values of $\left\langle S^{2}\right\rangle_{0}$ are equivalent, but for a copolymer chain these values are not usually equivalent. Scholte ${ }^{5}$ has derived an equation of optical $\left\langle S^{2}\right\rangle_{0}$ for block copolymers consisting of many blocks. Using distribution function theory ${ }^{11-14}$, the following equation for the optical $\left\langle S^{2}\right\rangle_{0}$ for copolymers is obtained.

$$
\left\langle S^{2}\right\rangle_{0}=\left(1 / M^{2} \nu^{2}\right) \sum_{0 \leq i<j \leq n}\left(\nu_{i} m_{i}\right)\left(\nu_{j} m_{j}\right)\left\langle r_{i j}^{2}\right\rangle_{0}
$$

where $\nu$ is the refractive index increment of a copolymer, and $\nu_{i}$ is that of structural unit $i$. Comparing eq 37 with eq 1 , we can calculate the optical $\left\langle S^{2}\right\rangle_{0}$ using the product of the refractive index increment and the mass of each structural unit instead of the mass. Thus, we can have a method which estimates $\left\langle S^{2}\right\rangle_{0}$ from light scattering data.

\section{APPENDIX}

\section{The Theorem of Lagrange}

A copolymer chain which is composed of $n+1$ structural units is shown in Figure 2. For this chain the center of mass of each structural unit deviates from the corresponding skeletal atom. The squared radius of gyration $S^{2}$ of this chain is represented by

$$
S^{2}=(1 / M) \sum_{i=0}^{n} \sum_{k=0}^{p_{i}} a_{i, k} S_{i, k}^{2}
$$

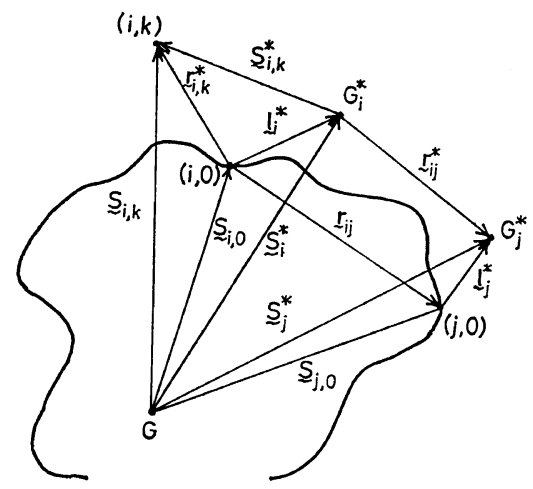

Figure 2. A copolymer chain model which has differences between the locations of the centers of mass of each structural unit and those of the corresponding skeletal atom.

where $S_{i, k}$ is the vector from $G$ (the center of mass of the whole chain) to the $k$-th atom of structural unit $i, i . e$., atom $(i, k)$. Using $S_{i}{ }^{*}$, which is the vector from $G$ to $\boldsymbol{G}_{i}{ }^{*}$, and $\boldsymbol{S}_{i, k}^{*}$, which is the vector from $G_{i}^{*}$ to atom $(i, k)$,

$$
\boldsymbol{S}_{i, k}=\boldsymbol{S}_{i}^{*}+\boldsymbol{S}_{i, k}^{*} .
$$

From the definition of $\boldsymbol{S}_{i, k}^{*}$, we obtain $\sum_{k=0}^{p i} a_{i, k} \boldsymbol{S}_{i, k}^{*}$ $=0$, Using these equations, eq $\mathrm{A} 1$ is converted into the following equation:

$$
S^{2}=(1 / M) \sum_{i=0}^{n} a_{i} S_{i}^{* 2}+(1 / M) \sum_{i=0}^{n} \sum_{k=0}^{p_{i}} a_{i, k} S_{i, k}^{* 2}
$$

The first term of this equation gives the squared radius of gyration when the mass of each structural unit concentrates on the center of mass of the corresponding structural unit. The second term gives the squared radius of gyration of a structural unit averaged over all the units.

Then, we represent the first term and the second term of eq A2 by $S^{* 2}$ and $S^{\prime 2}$, respectively. Using the vector from $G_{i}{ }^{*}$ to $G_{j}{ }^{*}$, i. e., $r_{i j}^{*}$, we obtain

$$
S^{* 2}=\left(1 / M^{2}\right) \sum_{0 \leq i<j \leq n} \sum_{i} a_{j} r_{i j}^{*^{2}}
$$

with

$$
\boldsymbol{r}_{i j}^{*}=\boldsymbol{r}_{i j}-\boldsymbol{l}_{i}^{*}+\boldsymbol{l}_{j}^{*}
$$

Substituting eq A4 into A3, we obtain

$$
\begin{aligned}
S^{* 2}= & \left(1 / M^{2}\right) \sum_{0 \leq i<j \leq n} a_{i} a_{j} r_{i j}^{2} \\
& +\left(1 / M^{2}\right) \sum_{0 \leq i<j \leq n} a_{i} a_{j} \boldsymbol{r}_{i j}\left(\boldsymbol{l}_{j}{ }^{*}-\boldsymbol{l}_{i}{ }^{*}\right)
\end{aligned}
$$




$$
\begin{aligned}
& -\left(2 / M^{2}\right) \sum_{0 \leq i<j \leq n} \sum_{i} a_{j} l_{i}{ }^{*} l_{j}{ }^{*} \\
& +(1 / M) \sum_{i=0}^{n} a_{i} l_{i}^{* 2}
\end{aligned}
$$

Using $\boldsymbol{r}_{i, k}^{*}$ which is the vector from the skeletal atom $(i, 0)$ to the atom $(i, k)$, we obtain

$$
\begin{aligned}
S^{\prime 2}= & (1 / M) \sum_{i=0}^{n} \sum_{k=0}^{p_{i}} a_{i, k} r_{i, k}^{* 2} \\
& -(1 / M) \sum_{i=0}^{n}\left(1 / a_{i}\right) \sum_{k=0}^{p_{i}} \sum_{h=0}^{p_{i}} a_{i, k} a_{i, h} r_{i, k}^{*} \boldsymbol{r}_{i, h}^{*}
\end{aligned}
$$

From the definition of $l_{i}{ }^{*}$,

$$
\boldsymbol{l}_{i}^{*}=\left(1 / a_{i}\right) \sum_{k=0}^{p_{i}} a_{i, k} \boldsymbol{r}_{i, k}^{*}
$$

Using eq A5, A6, and A7, we obtain eq A8 from A2.

$$
\begin{aligned}
S^{2}= & \left(1 / M^{2}\right) \sum_{0 \leq i<j \leq n} a_{i} a_{j} r_{i j}^{2} \\
& +\left(2 / M^{2}\right) \sum_{0 \leq i<j \leq n} a_{i} a_{j} \boldsymbol{r}_{i j}\left(\boldsymbol{l}_{j}{ }^{*}-\boldsymbol{l}_{i}{ }^{*}\right) \\
& -\left(2 / M^{2}\right) \sum_{0 \leq i<j \leq n} a_{i} a_{j} \boldsymbol{l}_{i}{ }^{*} \boldsymbol{l}_{j}{ }^{*} \\
& +(1 / M) \sum_{i=0}^{n} \sum_{k=0}^{p_{i}} a_{i, k} r_{i, k}^{* 2}
\end{aligned}
$$

Taking the statistical mechanical average over all configurations of this chain, we obtain the final result, i. e., eq 12.

\section{REFERENCES}

1. P. J. Flory, "Statistical Mechanics of Chain Molecules," John Wiley \& Sons, Inc., New York, N. Y., 1969.

2. P. J. Flory, Macromolecules, 7, 381 (1974).

3. W. G. Miller, D. A. Brant, and P. J. Flory, J. Mol. Biol., 23, 67 (1967).

4. S. Tanaka and A. Nakajima, Polym. J., 2, 725 (1971).

5. Th. G. Scholte, J. Polym. Sci., Part A-2, 5, 461 (1967).

6. W. L. Mattice, Macromolecules, 9, 48 (1976).

7. P. J. Flory, "Principles of Polymer Chemistry," Cornell University Press, Ithaca, N. Y., 1953.

8. C. Tanford, "Physical Chemistry of Macromolecules," John Wiley \& Sons, Inc., New York, N. Y., 1961.

9. H. Yamakawa, "Modern Theory of Polymer Solutions," Harper \& Row Publishers, New York, N. Y., 1971.

10. M. B. Huglin, "Light Scattering from Polymer Solutions," Academic Press, New York, N. Y., 1972.

11. B. H. Zimm, J. Chem. Phys., 16, 1093 (1948).

12. B. H. Zimm, ibid., 16, 1099 (1948).

13. A. C. Albrecht, ibid., 27, 1014 (1957).

14. H. Yamakawa, ibid., 46, 973 (1967). 